\title{
OPEN Single cell transcriptomics reveals the heterogeneity of the human cornea to identify novel markers of the limbus and stroma
}

\author{
Pere Català ${ }^{1,2}$, Nathalie Groen ${ }^{3}$, Jasmin A. Dehnen ${ }^{1,2}$, Eduardo Soares ${ }^{1,2}$, \\ Arianne J. H. van Velthoven ${ }^{1,2}$, Rudy M. M. A. Nuijts ${ }^{2}$, Mor M. Dickman ${ }^{1,2 \varpi} \&$ \\ Vanessa L. S. LaPointe ${ }^{1 \bowtie}$
}

The cornea is the clear window that lets light into the eye. It is composed of five layers: epithelium, Bowman's layer, stroma, Descemet's membrane and endothelium. The maintenance of its structure and transparency are determined by the functions of the different cell types populating each layer. Attempts to regenerate corneal tissue and understand disease conditions requires knowledge of how cell profiles vary across this heterogeneous tissue. We performed a single cell transcriptomic profiling of 19,472 cells isolated from eight healthy donor corneas. Our analysis delineates the heterogeneity of the corneal layers by identifying cell populations and revealing cell states that contribute in preserving corneal homeostasis. We identified expression of CAV1, HOMER3 and CPVL in the corneal epithelial limbal stem cell niche, CKS2, STMN1 and UBE2C were exclusively expressed in highly proliferative transit amplifying cells, CXCL14 was expressed exclusively in the suprabasal/superficial limbus, and NNMT was exclusively expressed by stromal keratocytes. Overall, this research provides a basis to improve current primary cell expansion protocols, for future profiling of corneal disease states, to help guide pluripotent stem cells into different corneal lineages, and to understand how engineered substrates affect corneal cells to improve regenerative therapies.

The healthy cornea is a transparent and avascular tissue that allows light to enter the eye and accounts for most of its refractive power. The cornea is composed of five layers: its outer surface is a stratified sheet of corneal epithelial cells that reside on the Bowman's layer, a collagen-based acellular membrane synthesized by the stromal keratocytes. The keratocytes populate the corneal stroma, the middle layer of the cornea that is composed of structured collagen fibers and other extracellular matrix proteins. The corneal endothelium forms a thin monolayer of tightly packed hexagonal cells that line in the innermost surface of the cornea and reside in close contact to the stroma on the Descemet's membrane.

Corneal structure and transparency are governed by the functions of the cell types populating each layer. Epithelial cells act as a biological barrier to block the passage of foreign material and provide a smooth surface that absorbs nutrients. Keratocytes maintain extracellular matrix homeostasis responsible for the cornea's biomechanical and optical properties, and endothelial cells serve as active pumps transporting ions, metabolites and fluid to maintain corneal hydration and transparency.

Selective replacement of dysfunctional single corneal layers with that of a donor ${ }^{1}$, the autologous transplantation of primary cultured corneal epithelial limbal stem cells ${ }^{2}$, and the treatment of endothelial dysfunctions with primary cultured allogeneic corneal endothelial cells ${ }^{3}$ are already a therapeutic reality. Gaining deeper understanding of corneal cell profiles and their transcriptomic signatures can be highly relevant for the improvement of such therapies.

In order to further understand the cellular complexity of this heterogeneous tissue, we provide a high-quality single-cell ribonucleic acid sequencing (scRNAseq) dataset from 19,472 corneal cells isolated from four female and four male donors. With it, we depict the diverse cell populations and provide a comprehensive cell atlas of

\footnotetext{
${ }^{1}$ Department of Cell Biology-Inspired Tissue Engineering, MERLN Institute for Technology-Inspired Regenerative Medicine, P.O. Box 616, 6200 MD Maastricht, The Netherlands. ${ }^{2}$ University Eye Clinic Maastricht, Maastricht University Medical Center+, PO Box 5800, 6202 AZ Maastricht, The Netherlands. ${ }^{3}$ Single Cell Discoveries, Utrecht, The Netherlands. ${ }^{\boxplus}$ email: mor.dickman@mumc.nl; v.lapointe@maastrichtuniversity.nl
} 


\begin{tabular}{|l|l|l|l|l|l|}
\hline Cornea & Sex & Age (years) & Preservation & Eye bank & Experiment \\
\hline 01 & Female & 62 & Optisol $\left(4^{\circ} \mathrm{C}\right.$ for 5 days $)$ & Saving Sight & $9.5 \mathrm{~mm} \emptyset$ trephined cornea scRNA-seq \\
\hline 02 & Male & 22 & Optisol $\left(4^{\circ} \mathrm{C}\right.$ for 6 days $)$ & Saving Sight & $9.5 \mathrm{~mm} \emptyset$ trephined cornea scRNA-seq \\
\hline 03 & Male & 75 & Optisol $\left(4^{\circ} \mathrm{C}\right.$ for 10 days $)$ & Saving Sight & $9.5 \mathrm{~mm} \emptyset$ trephined cornea scRNA-seq \\
\hline 04 & Female & 48 & Optisol $\left(4^{\circ} \mathrm{C}\right.$ for 8 days $)$ & Saving Sight & $9.5 \mathrm{~mm} \emptyset$ trephined cornea scRNA-seq \\
\hline 05 & Male & 75 & Organ culture media $\left(31^{\circ} \mathrm{C}\right.$ for 21 days $)$ & ETB-BISLIFE & $9.5 \mathrm{~mm} \emptyset$ trephined cornea scRNA-seq \\
\hline 06 & Female & 75 & Organ culture media $\left(31^{\circ} \mathrm{C}\right.$ for 15 days $)$ & ETB-BISLIFE & $9.5 \mathrm{~mm} \emptyset$ trephined cornea scRNA-seq \\
\hline 07 & Female & 71 & Organ culture media $\left(31^{\circ} \mathrm{C}\right.$ for 18 days $)$ & ETB-BISLIFE & Limbus scRNA-seq \\
\hline 08 & Male & 79 & Organ culture media $\left(31^{\circ} \mathrm{C}\right.$ for 14 days $)$ & ETB-BISLIFE & Limbus scRNA-seq \\
\hline 09 & Female & 78 & Organ culture media $\left(31^{\circ} \mathrm{C}\right.$ for 7 days $)$ & ETB-BISLIFE & Immunofluorescence \\
\hline 10 & Male & 71 & Organ culture media $\left(31^{\circ} \mathrm{C}\right.$ for 8 days $)$ & ETB-BISLIFE & Immunofluorescence \\
\hline
\end{tabular}

Table 1. Donor cornea information.

the healthy human cornea. The transcriptomic cell census identified subpopulations with different roles in the maintenance of corneal homeostasis and provides a baseline to improve primary cell expansion protocols, for future profiling of corneal disease states, to help guide pluripotent stem cells into different corneal lineages, and to understand how engineered substrates affect corneal cells to improve regenerative therapies. Furthermore, this dataset identified markers exclusively expressed in cells comprising the limbal epithelial stem cell niche, highly proliferating epithelial cells, and stromal keratocytes which could be used as reference to improve current corneal cell replacement therapies.

\section{Results}

Five major cell clusters were identified in the corneal tissue. Six donor corneas and the limbi of two donor corneas (Table 1) were manually dissected and dissociated into single cells for single-cell RNA sequencing. After filtering low quality cells, the transcriptome profiles of 19,472 cells were further analyzed (Fig. 1a).

Data of the 19,472 sequenced cells were embedded in a uniform manifold approximation and projection (UMAP) and unbiased low resolution clustering revealed five major cell clusters (Fig. 1b, left). The expression of specific corneal layer marker (keratin 5 (KRT5) for epithelium ${ }^{4}$, keratocan (KERA) for stromal cells ${ }^{5}$, and transporter-like protein 11 (SLC4A11) for endothelium ${ }^{6}$ ) identified the three main layers within the five identified clusters (Fig. 1c). Differential gene expression profiling of the clusters further confirmed the identification of a corneal epithelial cell cluster comprising 5964, a corneal stromal cell cluster comprising 12,344 cells, a corneal endothelial cell cluster comprising 842 cell, and non-corneal clusters of blood/lymphatic vessel endothelial cells comprising 36 cells, and immune cells comprising 216 cells (Fig. 2). Cell clusters from the three corneal cell layers were further classified separately at higher resolution in different corneal layer-specific subclusters (Fig. 1b, right).

Nine cell clusters were identified within the corneal epithelium. A major cluster of 5964 corneal epithelial cells was identified and further analysis revealed nine subclusters (E0-8, Fig. 3a). Differential gene expression profiling was used for further identification of the subclusters. Cluster E3 presented a high expression of stromal cell markers KERA, lumican (LUM), and aldehyde dehydrogenase 3 member A1 (ALDH3A1) $)^{5,7,8}$, as well as a reduced expression of corneal epithelial marker keratin $12(K R T 12)^{9,10}$ compared to the other epithelial subclusters (Fig. 3b and Supplementary Figure S1). Putative doublet analysis of cluster E3 suggested epithelialstromal keratocyte cell doublets (Supplementary Figure S2). Cluster E7 was identified as conjunctival epithelial cells based on high expression of conjunctival markers such as keratin 13 (KRT13), keratin 15 (KRT15), and keratin 19 (KRT19) $^{11,12}$, and the low expression of the corneal epithelial marker KRT12 (Fig. 3b and Supplementary Figure S1).

Cells forming clusters E6, E8 and E8 showed increased expression of corneal limbal markers keratin 14 $\left(K_{R T 14}\right)^{13,14}, K R T 15^{15,16}$, and S100 calcium binding protein A2 (S100A2) ${ }^{17}$ compared to the other identified clusters suggesting their location in the corneal limbus (Fig. $3 \mathrm{~b}$ and Supplementary Figure S1). Interestingly, cluster E6 showed an increased expression of the superficial epithelium limbus marker ${ }^{17} \mathrm{~S} 100$ calcium binding protein A8 (S100A8) compared with clusters E0 and E8 and a reduced expression of the basal corneal epithelial cell markers connexin 26 (GJB2), connexin 30 (GJB6), and integrin $\beta 4$ (ITGB4) $)^{18-20}$, (Fig. 3b and Supplementary Figure S1) leading to its identification as a population of wing/superficial epithelial cells in the limbus or peripheral cornea. Cluster E0 presented an increased expression of GJB6, and GJB2, which are predominantly found in basal corneal epithelium ${ }^{18}$ suggesting these cells formed a basal corneal epithelial cell population in the limbal stem cell niche or peripheral cornea (Fig. $3 \mathrm{~b}$ and Supplementary Figure S1). Finally, the high expression of mitogenic factors Ki-67 (MKI67) $)^{21}$, survivin $(B I R C 5)^{22}$ and H2A histone family member X $(H 2 A F X)^{23}$ in cluster E8, as well as the differential expression of transit-amplifying cell marker $\mathrm{CD} 109^{24}$, suggested that cluster E8 was formed by highly proliferative transit-amplifying cells in the limbal stem cell niche or peripheral cornea (Fig. $3 \mathrm{~b}$ and Supplementary Figure S1). No quiescent limbal epithelial stem cells expressing ABCB5, ABCG2 and $C D 200^{24-27}$ were identified in this dataset (Supplementary Figure S1).

Cluster E5 presented high expression of GJB6 and ITGB4 associated with basal epithelium ${ }^{18}$ and KRT12, and $K R T 3$ associated with terminally differentiated corneal epithelium ${ }^{27-29}$, along with a reduced expression of KRT14 
a

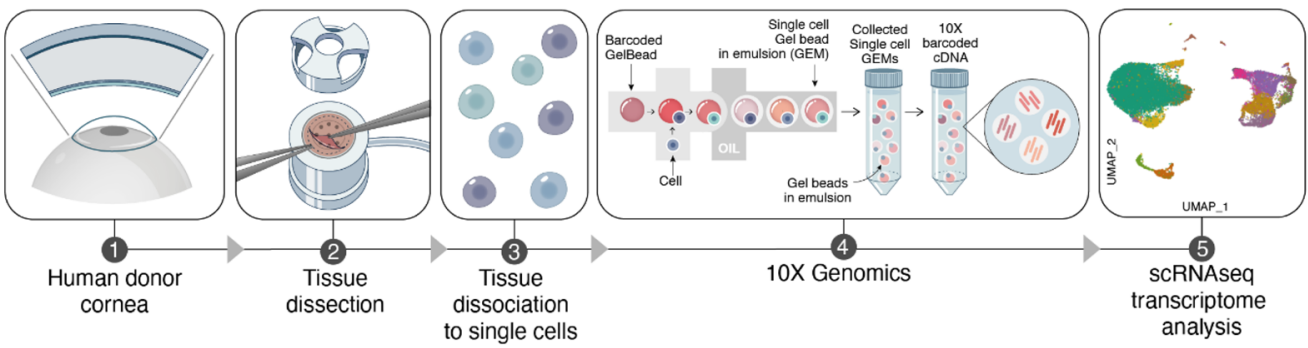

b

Blood/lymphatic 。 vessel endothelium Immune cells
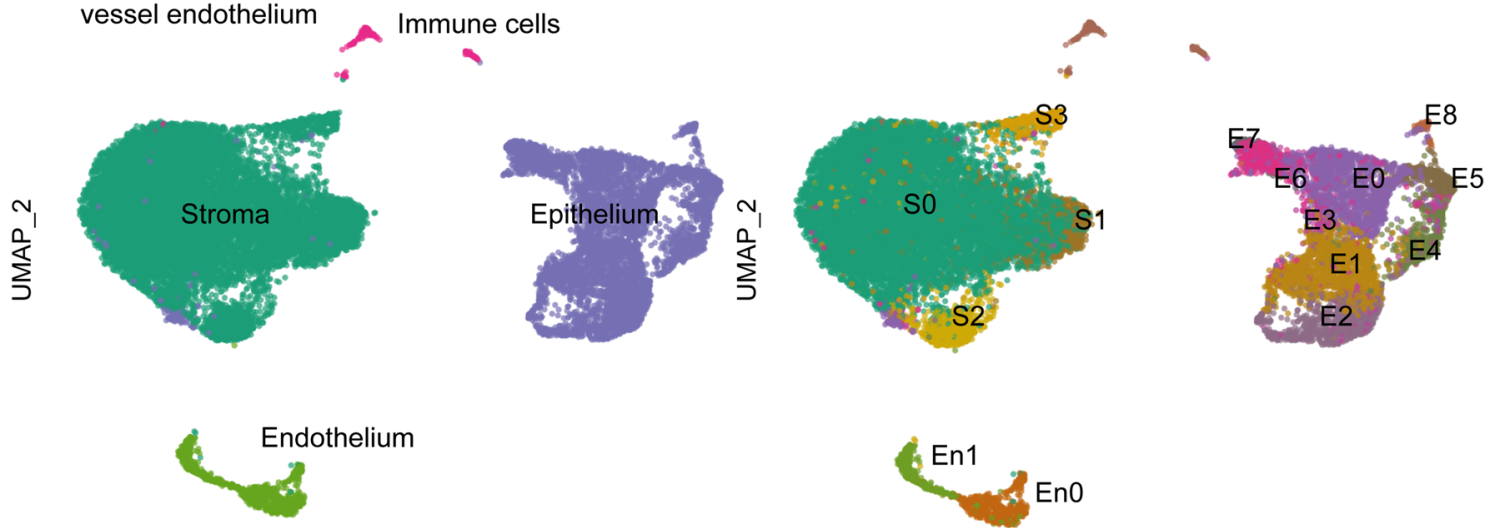

UMAP_1

UMAP_1

C
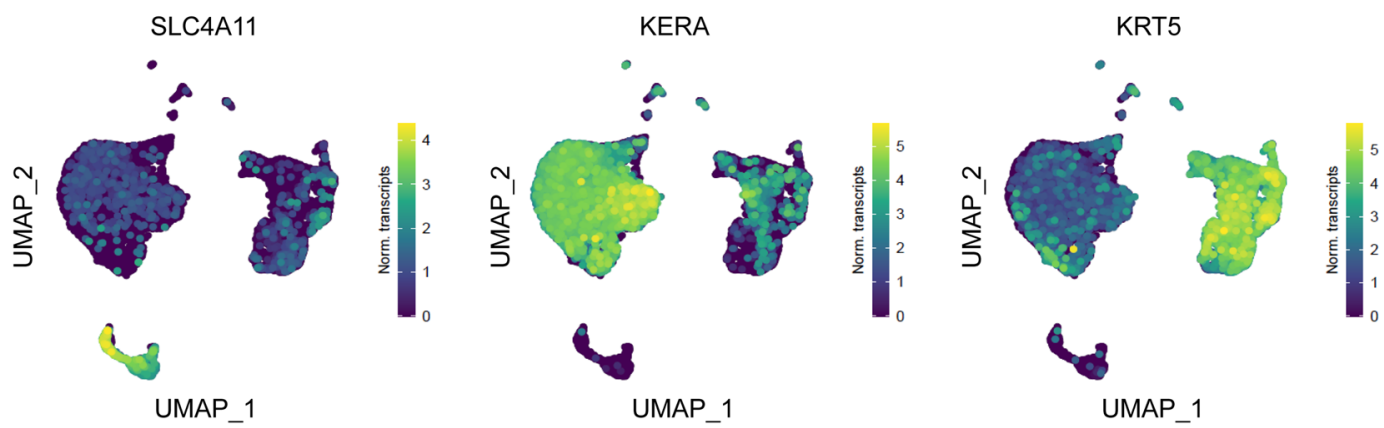

Figure 1. Cell clusters from all three corneal layers and two additional clusters of immune and blood/lymphatic endothelial cells were identified with scRNAseq analysis. Experimental overview for scRNAseq (a). UMAP of the 19,472 sequenced cells with the identified five major cell clusters (b, left). Cell clusters from the three corneal cell layers were further subclustered in different cell subpopulations (b, right). Single cell expression level UMAP of specific corneal layer markers supports cell cluster identification (c): SLC4A11 is corneal endothelial-specific, KERA is corneal stromal-specific, and KRT5 is corneal epithelial-specific.

compared to clusters E6, E8, and E0, and no expression of KRT15, suggesting these cells were corneal epithelial basal cells originating from the limbus (Fig. 3b and Supplementary Figure S1). Cluster E1 was identified as post-mitotic and terminally differentiated migratory epithelial cells based on the expression of genes associated with cell migration such as $R H O V^{30}$ and tight junction formation and obliteration $C L D N 7^{31}$ together with a high expression of corneal epithelial cell markers KRT12, KRT3, and KRT5. This cluster retained a low expression level of KRT14, implying their limbal origin (Fig. 3b and Supplementary Figure S1).

Cluster E4 was composed of cells with a high expression of KRT12, KRT3, and KLF5 suggesting this cluster was terminally differentiated cells from the central corneal epithelium (Fig. 3b and Supplementary Figure S1). The cells forming cluster E2 presented a high expression of KRT12, KRT24, and CXCL17 associated with wing/ superficial central epithelium (Fig. 3b and Supplementary Figure S1).

scRNAseq reveals novel specific markers for the corneal limbal stem cell niche and transit-amplifying cells. Differential gene expression analysis of the basal corneal limbal epithelial cells (cluster $\mathrm{E} 0$ ), and the transit-amplifying cells (cluster E8), showed that genes encoding for caveolin-1 (CAV1), probable serine carboxypeptidase (CPVL), homer scaffolding protein 3 (HOMER3), and C-X-C motif chemokine 14 (CXCL14) were highly expressed in both clusters (Fig. 3c), suggesting they could be markers of the human limbal stem cell niche. Moreover, the expression of the markers highly correlated in clusters E0 and E8 (Fig. 3d), again 


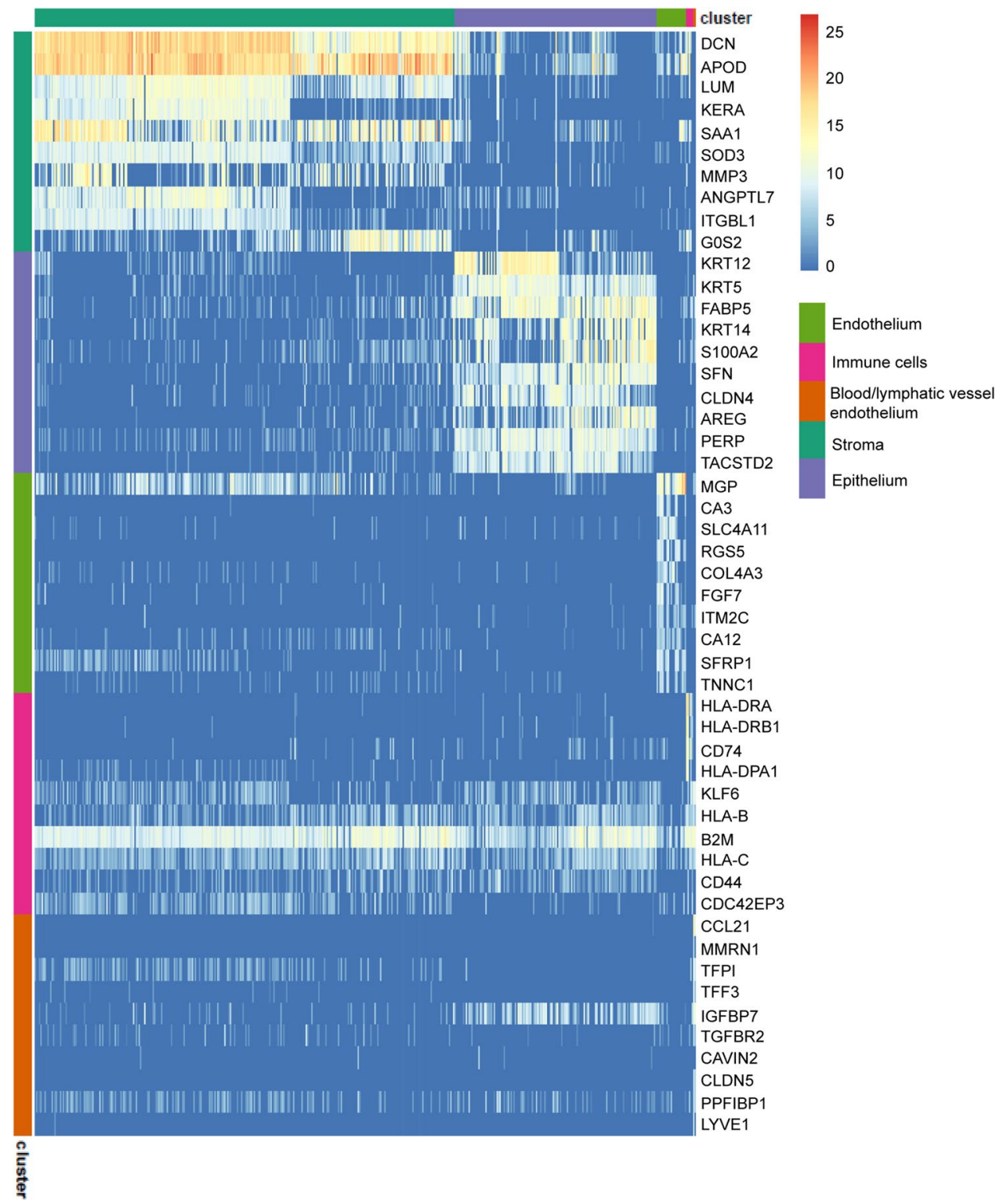

Figure 2. The heatmap of the top 10 differentially expressed genes for each cluster showed distinct transcriptomic profiles for the five major cell clusters and allowed cell cluster identification.

suggesting their identity of corneal epithelial cells located in the limbal stem cell niche. Interestingly, a cluster of basal corneal epithelial cells (cluster E5) retained some expression of the identified markers, in line with the limbal origin annotated for this cluster.

To confirm these findings, expression of the markers in the corneal epithelial limbus and central cornea was assessed by immunofluorescence (Fig. 4 and Supplementary Figure S3). Caveolin-1 and CXCL14 were expressed in the limbus of the cornea, whereas caveolin-1 was minimally expressed and CXCL14 absent in the central cornea (Fig. 4 and Supplementary Figure S3). Interestingly, CXCL14 was expressed in the suprabasal/superficial limbus and not in the limbal stem cell niche. Central basal corneal epithelial cells retained minimal expression of both $\Delta$ Np63 and caveolin-1 (Fig. 4 and Supplementary Figure S3), suggesting their limbal origin. In contrast, HOMER3 and CPVL were expressed both in the limbus and in the central cornea, where central basal epithelial cells appeared to have higher expression (Fig. 4 and Supplementary Figure S3). Caveolin-1 expression was further validated on human primary cultured corneal limbal epithelial cells, where it was expressed in $\Delta$ Np63-positive limbal epithelial stem cells (Supplementary Figure S4).

Differential gene expression analysis on the transit-amplifying cells (cluster E8) revealed that the expression of CKS2, STMN1, and UBE2C was exclusive to this cluster. Expression of cyclin-dependent kinase 2, stathmin-1, and ubiquitin conjugating enzyme E2 C, was exclusive to the limbus and absent in the central cornea (Fig. 4, Supplementary Figure S3, and Supplementary Figure S5). Cyclin-dependent kinase 2 was further validated on human primary cultured corneal limbal epithelial cells, where it was expressed in in p63a-positive limbal epithelial stem cells (Supplementary Figure S6). 
a

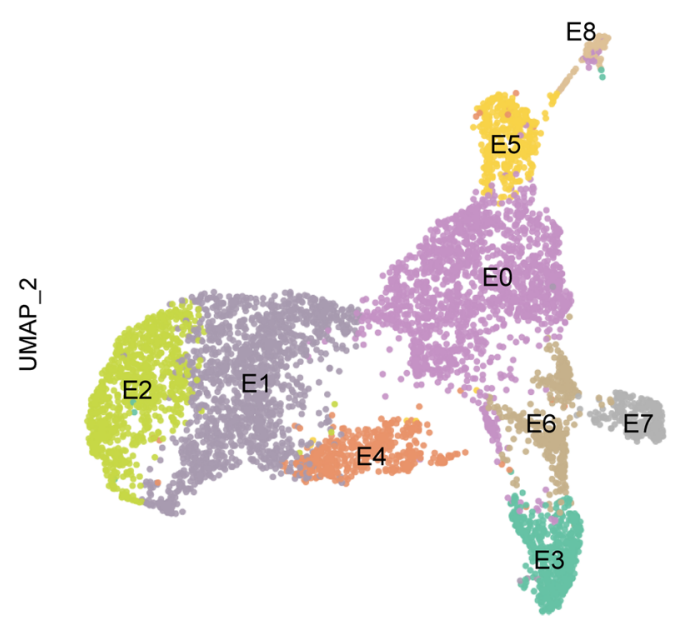

UMAP_1

C
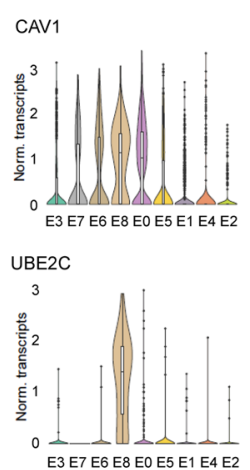

b
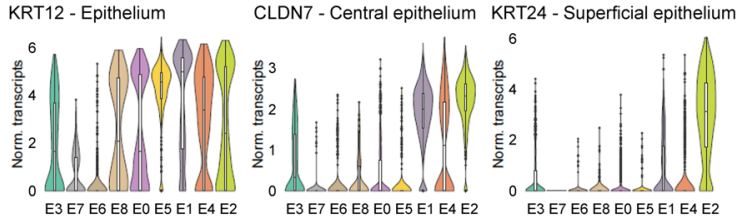

GJB6 - Basal/wing epithelium ITGB4 - Basal epithelium
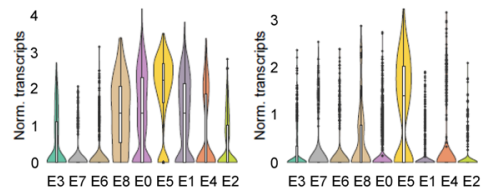

MKI67 - Proliferation
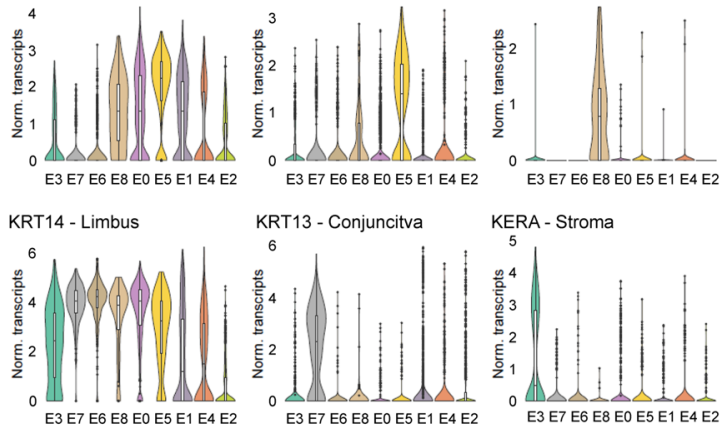

KERA - Stroma

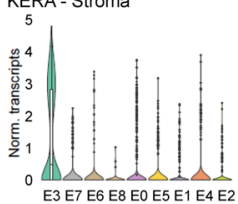

CXCL14

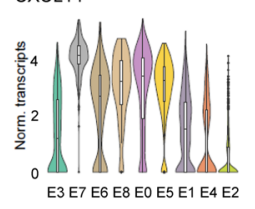

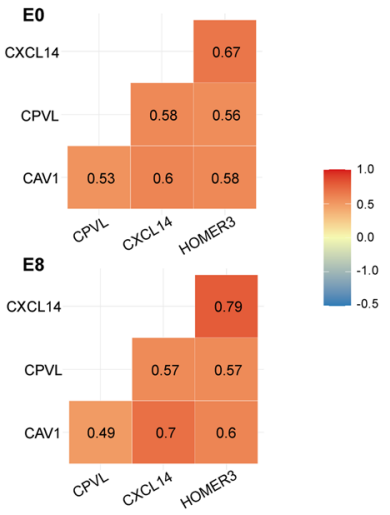

Figure 3. A major cluster of 5964 corneal epithelial cells was identified with scRNAseq analysis. The UMAP revealed nine different cell subclusters: a cluster of basal limbal epithelial cells (E0), a cluster of migratory epithelial cells (E1), a cluster of wing/superficial epithelial cells (E2), a cluster of stromal-epithelial doublets (E3), a cluster of central epithelial cells (E4), a cluster of basal central epithelial cells originating from the limbus (E5), a cluster of wing epithelial cells in the limbus (E6), a cluster of conjunctival cells (E7), and a cluster of highly proliferative transit-amplifying cell (E8) (a). Violin plots show marker genes for corneal epithelium (KRT12), central (CLDN7), superficial (KRT24), suprabasal (GJB6), basal (ITGB4), and limbal (KRT14) corneal epithelium, as well as proliferation (MKI67), conjunctival (KRT13) and stromal (KERA) markers, for the identification of cell subclusters (b). Differential gene expression identified novel epithelial limbal niche-specific markers and proliferative epithelial cell-specific markers, and are visualized as violin plots (c). Correlation analysis confirmed the co-expression of limbal niche-specific markers in peripheral/limbal epithelial cell populations (d).

Four cell clusters were identified within the corneal stroma. A major cluster of 12,344 corneal stromal cells was identified, and further analysis revealed four different subclusters of corneal stromal cells (S0-S3, Fig. 5a). High expression of stromal keratocyte markers LUM, KERA, or ALDH3A $1^{5,7,8}$ suggested that clusters S0, $\mathrm{S} 1$ and S2 were stromal keratocytes (Fig. 5b). Cluster S2 showed increased expression of extracellular matrix proteins such as COL12A1,COL6A2, COL6A1, and LAMB2 suggesting these were activated keratocytes that played a crucial role in maintaining the corneal stromal extracellular matrix (Fig. 5b and Supplementary Figure S7). Cluster S3 showed a decreased expression of keratocyte markers LUM, KERA, and ALDH3A1 and increased expression of the fibroblastic marker $C D 44^{32-34}$ compared to clusters S0, S1 and S2 (Fig. 5b and Supplementary Figure S7). The myofibroblast-specific marker, $\alpha$-smooth muscle actin $(A C T A 2)^{35,36}$, was not detected in cluster S3 (Supplementary Figure S7), suggesting it is composed of keratocytes that are in transition to stromal myofibroblasts.

Finally, differential gene expression analysis of the major stromal cluster compared to the clusters associated with the corneal epithelial and endothelial layers identified that the expression of nicotinamide N-methyltransferase (NNMT) was exclusive to the corneal stromal cluster, suggesting that this gene could be used as a novel corneal stromal marker (Fig. 5b,c). 


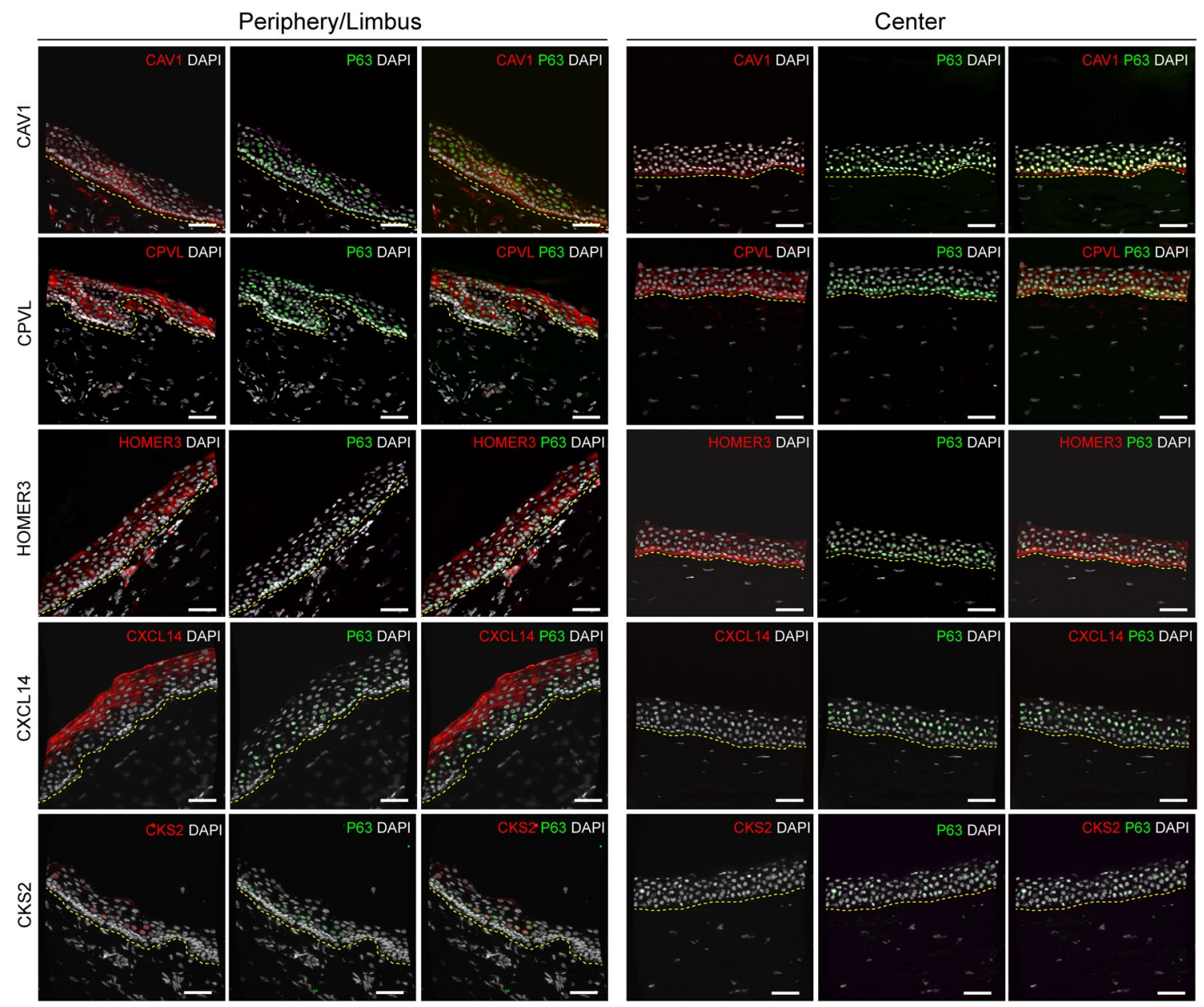

Figure 4. Immunofluorescence of caveolin-1 (CAV1), CXCL14 and CKS2 (red) on human corneal tissue cryosections confirmed differential protein expression in the limbus/periphery, and absence (CKS2, and CXCL14) or minimal expression (caveolin-1) in the central cornea. Basal corneal epithelial cells retained minimal expression of both $\triangle \mathrm{Np} 63$ and caveolin-1, suggesting their limbal origin. HOMER3 and CPVL were expressed in the limbus, but also in the central cornea, where central basal epithelial cells appeared to have higher expression. P63 ( $\Delta$ Np63 or p63a) was used as a corneal epithelial limbal cell marker (green). Cell nuclei were stained with DAPI (white). The yellow dashed line indicates the boundary between epithelium (above) and stroma (below). Scale bars represent $50 \mu \mathrm{m}$.

Two cell clusters were identified within the corneal endothelium. A major cluster of 842 corneal endothelial cells was identified and further analysis revealed two different subclusters of corneal endothelial cells (En0-1, Fig. 6a). Both clusters showed expression of corneal endothelial cell markers CD166 (ALCAM) and sPrdxl (PRDX1) 37,38 and functional markers $\mathrm{Na}^{+} / \mathrm{K}^{+}$ATPase $(A T P 1 A 1)^{6}$ and sodium bicarbonate transporter-like protein $11(S L C 4 A 11)^{6}$ confirming the corneal endothelial phenotype (Fig. $6 \mathrm{~b}$ and Supplementary Figure S7). Differential expression analysis revealed that cluster En0 possessed a lower expression of tight junction protein zona occludens- $1(T J P 1)^{6}$ and focal adhesion regulator microtubule-actin cross-linking factor-1 $(M A C F 1)^{39}$ compared to cluster En1 (Fig. 6b), suggesting cells in cluster En0 could preferentially migrate upon corneal endothelial damage to contribute to tissue repair. Furthermore, cluster En 1 possessed a higher expression of COL4A3 (Fig. 6b) suggesting these cells could play an important role on Descemet's membrane homeostasis. Interestingly, both clusters retained expression of PITX2, a periocular mesenchyme marker associated with progenitor endothelial cells ${ }^{40,41}$. No endothelial fibroblasts were identified, evidenced by the absence of ACTA2 and CD44 (Supplementary Figure S8).

\section{Discussion}

Gaining transcriptomic information at the single cell level of human corneal cells enables a greater understanding of this heterogeneous tissue. In this study, we have performed a scRNAseq analysis of the healthy cornea to create a comprehensive cell atlas of the human cornea (Fig. 7). Moreover, scRNAseq analysis enabled the identification of novel markers of the limbal epithelial stem cell niche, transit-amplifying cells, and stromal keratocytes. The data generated can serve as a reference cell atlas with a major impact in the further improvement and development of cell replacement therapies or regenerative medicine approaches for treating corneal 
a

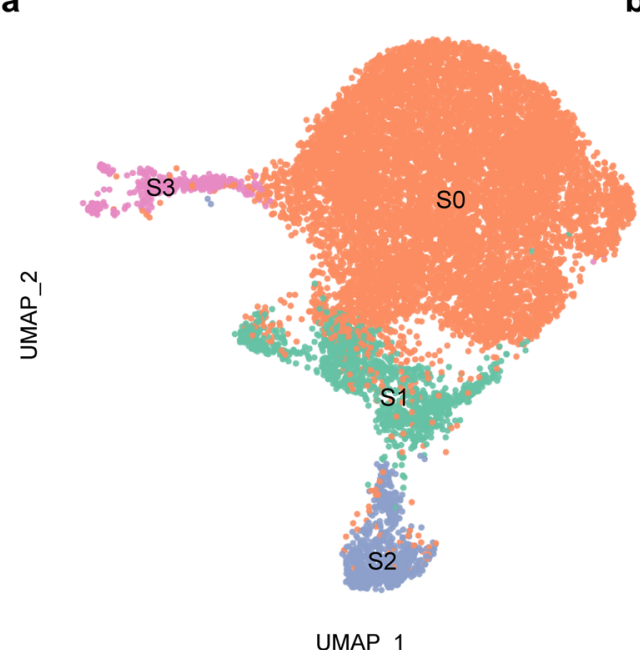

b

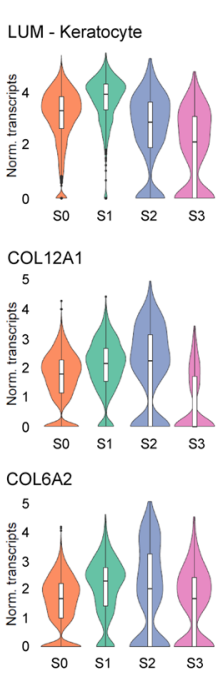

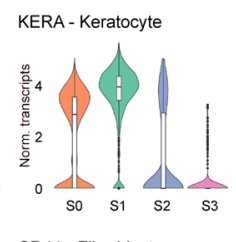

CD44 - Fibroblast

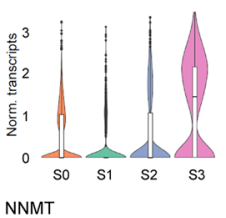

NNMT

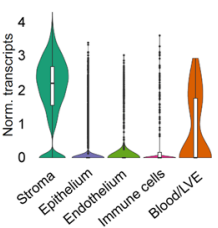

C

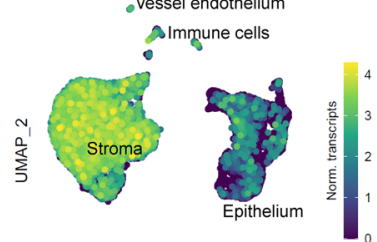

Figure 5. A major cluster of 12,344 corneal stromal cells was identified with scRNAseq analysis. The UMAP revealed four different cell subclusters, three keratocyte clusters (S0-S2), with one having a high extracellular matrix protein secretion profile (S2) and a cluster of keratocytes transitioning to stromal myofibroblasts (S3) (a). Violin plots show marker genes for stromal keratocytes (KERA and $L U M)$, stromal fibroblasts (CD44), collagen (COL6A2 and COL12A1) secretion for the identification of cell subclusters, and NNMT expression exclusive to the stroma within the cornea (b). Single cell expression level UMAP of NNMT further confirms differential expression of NNMT in the stromal cluster (c).

a

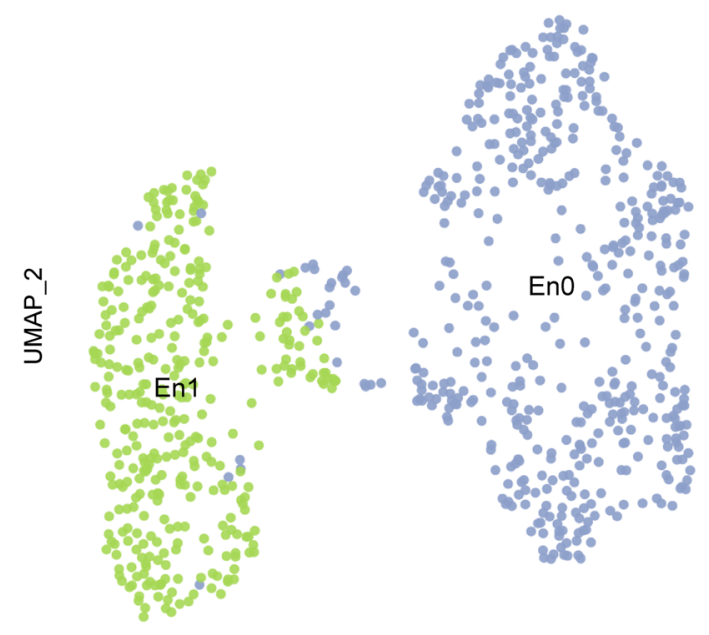

UMAP_1 b
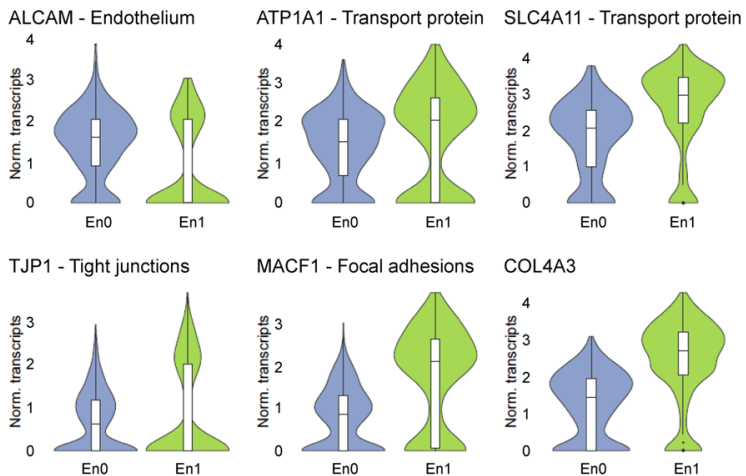

MACF1 - Focal adhesions

COL4A3

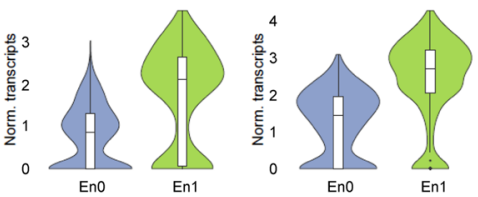

PITX2 - Periocular mesenchyme

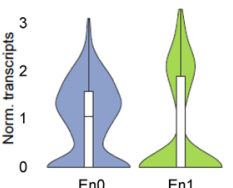

Figure 6. A major cluster of 842 corneal endothelial cells was identified with scRNAseq analysis. The UMAP revealed two different endothelial cell subclusters, a cell cluster with high collagen synthesis (En1) and another cell cluster with low tight junction and focal adhesion protein expression (En0) (a). Violin plots show marker genes for corneal endothelium (ALCAM), ion and bicarbonate transporters (ATP1A1 and SLC4A11 respectively), tight junction proteins (TJP1), focal adhesion protein regulator ( $M A C F 1)$, collagen secretion (COL4A3) and periocular mesenchyme (PITX2) (b).

blindness. This research further complements scRNAseq analysis of the developing human cornea ${ }^{42}$ and of the human corneal limbus ${ }^{43,44}$.

The corneal epithelium appeared to be the most heterogeneous corneal layer with 9 identified cell clusters. The transcriptomic signature of epithelial cells from the basal, wing and superficial layers of both central and limbus/peripheral cornea were identified. A population of conjunctival epithelial cells was detected (cluster E7; Fig. 3), likely representing contamination from the dissection process. Moreover, a cluster of highly proliferative 

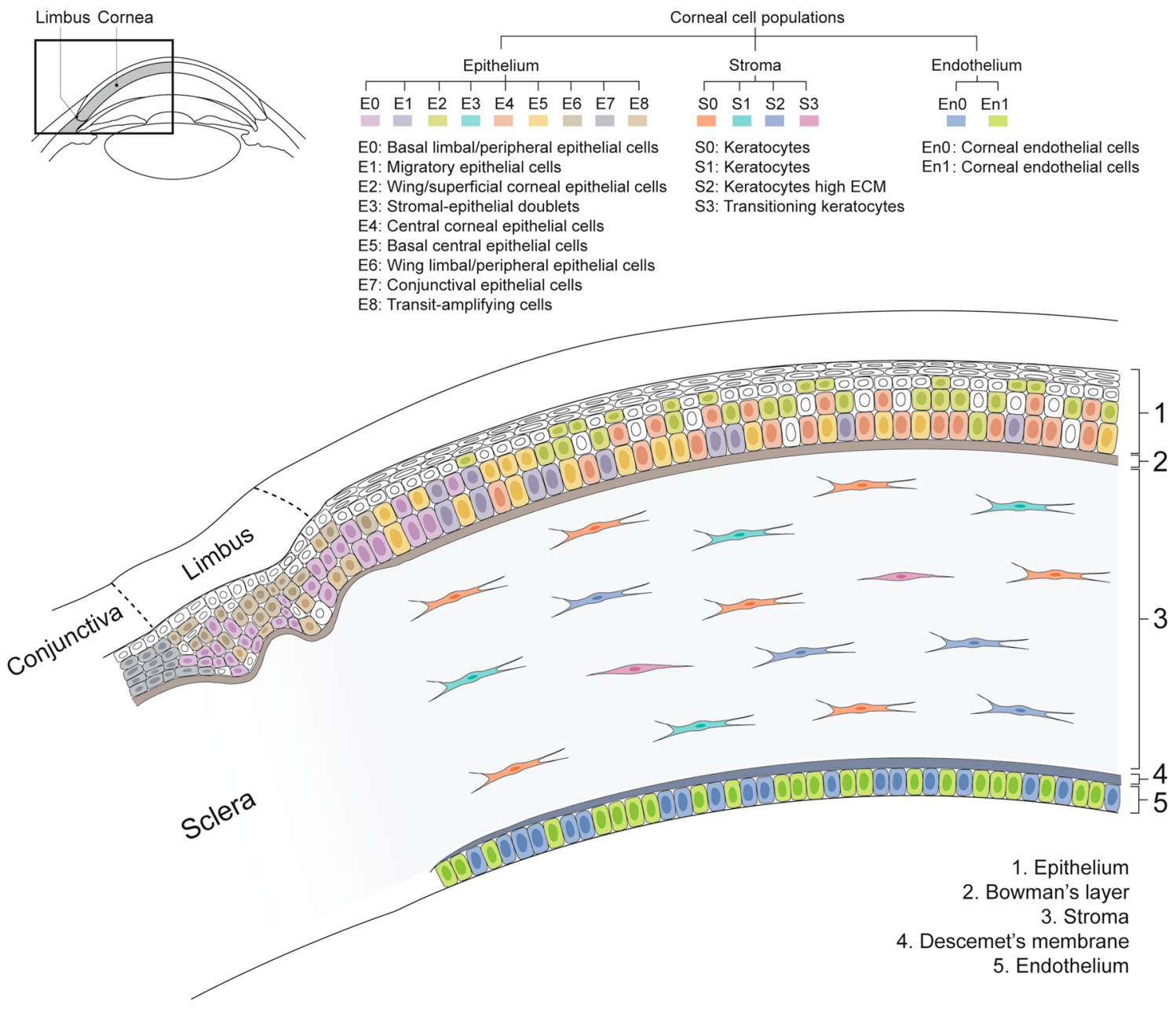

Figure 7. scRNAseq data analysis identified nine epithelial, four stromal and two endothelial corneal cell clusters. This figure presents a summary of the cell type and location of each cluster in the healthy human cornea, generating a comprehensive cell census of the healthy human cornea. In the epithelium: a cluster of basal limbal epithelial cells (E0), a cluster of migratory epithelial cells (E1), a cluster of wing/superficial epithelial cells (E2), a cluster of stromal-epithelial doublets (E3), a cluster of central epithelial cells (E4), a cluster of basal central epithelial cells originating from the limbus (E5), a cluster of wing epithelial cells in the limbus (E6), a cluster of conjunctival cells (E7), and a cluster of highly proliferative transit-amplifying cell (E8). In the stroma: three keratocyte clusters (S0-S2), with one having a high extracellular matrix protein secretion profile (S2) and a cluster of keratocytes transitioning to stromal myofibroblasts (S3). In the endothelium: a cell cluster with high collagen synthesis (En1), and a cell cluster with low tight junction and focal adhesion protein expression (En0).

transit-amplifying cells (cluster E8) was detected. No quiescent limbal epithelial stem cells expressing $A B C B 5$, TCF4, CD200 and ABCG2 were identified (Supplementary Figure S1). This result is in line with the study of $\mathrm{Li}$ et al., where out of 16,360 cells specifically isolated from the adult corneal limbus region, only 69 cells were identified as limbal epithelial stem cells, corresponding with $0.4 \%$ of the total sequenced cells ${ }^{43}$. Because our data present sequencing information of 5964 corneal epithelial cells, it is possible that there might not have been enough sequencing depth to detect such a rare population. Moreover, and in line with these results, in a recent study by Dou et al., expression of ABCB5 and ABCG2 was also minimally detected 47,627 cells isolated from the human corneal limbus, and was also associated to limitations in the sequencing depth ${ }^{44}$. These are opposing findings to the results presented by Collin et al. 2021, where a major cluster of 893 (4\%) limbal progenitor/stem cells was detected in a pool of 21,343 sequenced cells isolated from corneoscleral buttons ${ }^{42}$.

Differential analysis identified the expression of CAV1, HOMER3, CXCL14, and CPVL to be exclusive to cell clusters comprising the corneal epithelial stem cell niche (Fig. 3). In line with our findings, Collin et al. recently reported the exclusivity of CXCL14 and CPVL to the corneal epithelial stem cell niche ${ }^{42}$. Immunofluorescence analysis confirmed the expression of caveolin-1 and CXCL14 in the limbus, and their absence in the central cornea (Fig. 4). Nevertheless, CXCL14 was expressed in the superficial layers of the limbus but not the limbal stem cell niche. HOMER3 and CPVL were both expressed in the limbus, and in the central cornea, where central basal epithelial cells appeared to retain higher expression of these markers (Fig. 4). We further validated the finding of caveolin-1 with immunofluorescence in primary cultured limbal stem cells (Supplementary Figure S4). These results suggest that caveolin-1 could be used for the identification of epithelial limbal stem cells, with the 
advantage that it is a cell membrane marker, whereas p63 is expressed in the nucleus. This could open the door to isolating and enriching these cells for regenerative therapies. Furthermore, our study also revealed novel makers specific to transit-amplifying cells, namely CKS2, STMN1, and UBE2C. We further validated these findings with immunofluorescence, and confirmed their expression in the limbus and periphery of human corneas. Furthermore, expression of CKS2 was also assessed in primary cultured limbal stem cells (Supplementary Figure S6), suggesting this is a suitable marker for identifying highly proliferative transit-amplifying cells. Interestingly, primary limbal cells expressing a lower level amount of $\Delta \mathrm{Np} 63$ and $\mathrm{p} 63 \alpha$, based on fluorescence intensity, also showed a reduced expression of caveolin-1 and cyclin-dependent kinase 2 respectively (Supplementary Figures S4 and S6). Interestingly, two recent studies also reported STMN1 and UBE2C to be specific markers of limbal transit amplifying cells ${ }^{43,45}$. Finally, in a 2019 single cell transcriptome study, Kaplan et al. hypothesized that the stemness of mouse limbal epithelial stem cells could be regulated through autophagy ${ }^{46}$. The identification of caveolin-1, a facilitator of caveolin-mediated endocytosis, as a marker of the human corneal limbus in our study could support this hypothesis. Nevertheless, further research is required to confirm this theory.

It is important to highlight that as previously reported, the corneal epithelium sheds superficial layers when corneas are preserved in media ${ }^{47}$ and it is very likely that this cell atlas does not fully portray the most superficial layer of corneal epithelial cells. Two types of cornea preservation conditions, namely in Optisol-GS medium and organ culture medium, were used for this research, in line with clinical practice in the United States of America and Europe, respectively. No major differences in cell clustering were found between preservation conditions.

Three clusters of the corneal stroma were identified as corneal keratocytes (clusters S0, S1 and S2; Fig. 5). Cluster S2 was identified as cells with a key role in extracellular protein secretion to maintain the homeostasis of the stroma. Furthermore, we identified a cluster (S3) of keratocytes transitioning to myofibroblasts, though a fully differentiated myofibroblast phenotype expressing alpha smooth muscle actin was not identified. Interestingly, our analysis did not identify a population of corneal stromal stem cells expressing $A B C B 5$ and $A B C G 2$, as previously identified by Funderburgh and colleagues ${ }^{48-50}$. Our hypothesis is that the expression of these genes is induced by the primary expansion of corneal keratocytes and is not found in the cornea ${ }^{48-50}$. Finally, differential gene expression analysis identified that the expression of NNMT was exclusive to the corneal stroma, suggesting this could be used as a novel marker to identify corneal stromal cells. Interestingly, the study by Li et al. reported differential expression of NNMT in a cluster of 69 quiescent limbal stem cells expressing ABCB5 and ABCG2 ${ }^{43}$. As previously mentioned, perhaps due to limitations in sequencing depth, we did not find a population of limbal stem cells expressing ABCB5 and ABCG2, and therefore unable to confirm these results. Since Li and colleagues did not include a population of corneal stromal cells in their differential analysis, it also explains why NNMT was not detected as a stromal marker in their study. It is possible that NNMT is expressed specifically in both $\mathrm{ABCB}^{+} / \mathrm{ABCG}^{+}$quiescent limbal stem cells and in corneal stromal cells.

Two cell clusters were identified forming the corneal endothelium (Fig. 6). Both clusters showed high expression of corneal endothelial markers such as CD166 (ALCAM), sPrdx1 (PRDX1), ZO-1 (TJP1), or SLC4A11, confirming the endothelial phenotype. Nevertheless, expression of $C D 200$, reported as corneal endothelial cell marker in a previous study ${ }^{51}$, was not detected in our dataset, suggesting it might not be a specific marker for corneal endothelial cells. Despite sharing great similarity, one of the clusters (En1) expressed more extra cellular matrix proteins, suggesting that these cells could play a crucial role on maintaining the Descemet's membrane. Cell cluster En0 showed lower expression of tight junction proteins and focal adhesions, suggesting these cells could preferentially migrate upon corneal endothelial damage and contribute to tissue repair via migration or cytosolic expansion. Interestingly, our study did not detect a population of precursor endothelial cells, as discussed in previous studies ${ }^{52,53}$. Nevertheless, both corneal endothelial cell clusters detected retained expression of PITX2, a marker associated with neural crest-derived corneal endothelial cell precursors ${ }^{40,41}$. These data suggests that it is highly possible that a progenitor-like state is not exclusive to the peripheral corneal endothelium but to cells across the endothelium.

Interestingly, no corneal endothelial fibroblasts were detected in this study, indicated by the lack of expression of fibroblastic markers $\alpha$-smooth muscle actin (ACTA2) and CD44 (Supplementary Figure S8), which is in contrast to a previous report ${ }^{42}$. It is likely that the bulk enzymatic corneoscleral tissue desegregation performed by Collin et al., affected the susceptible corneal endothelial cells, causing endothelial cells transcriptomic bias portrayed in scRNAseq dataset, as showed in other studies ${ }^{54,55}$. We performed a more gentle approach to obtain single endothelial cells for sequencing, first dissecting the tissue and mechanically stripping the endothelium from the cornea, and then treating the endothelial cells with a shorter enzymatic digestion.

Overall, this study provides significant information to help understand the heterogeneity of the healthy human cornea, as well as understanding the gene expression stratification across cells present in the same corneal layer, while providing novel markers to identify specific cell types. Moreover, this transcriptomic cell atlas offers a baseline for future studies with the aim of regenerating corneal tissue or further developing corneal cell replacement therapies.

\section{Materials and methods}

Ethical statement. This study was performed in compliance with the tenets of the Declaration of Helsinki. Ten human donor corneas (Table 1) deemed unsuitable for transplantation were obtained from the Saving Sight Eye Bank (Kansas City, MO, USA) and the ETB-BISLIFE Multi-Tissue Center (Beverwijk, the Netherlands) were used for this study. Both male and female donor corneas, with ages ranging from 22 to 79 years and preserved either in Optisol-GS at $4{ }^{\circ} \mathrm{C}$ or in Organ culture media at $31{ }^{\circ} \mathrm{C}$ were used. Organ culture media comprised the following: minimum essential medium supplemented with $20 \mathrm{mM}$ HEPES, $26 \mathrm{mM}$ sodium bicarbonate, $2 \%$ (v/v) newborn calf serum (Thermo Fisher Scientific), $10 \mathrm{IU} / \mathrm{mL}$ penicillin, $0.1 \mathrm{mg} / \mathrm{mL}$ streptomycin and $0.25 \mu \mathrm{g} /$ 
$\mathrm{mL}$ amphotericin. The tissues used for scRNAseq had no history of ocular disease, chronic systemic disease or infection such as HIV or hepatitis B.

Manual dissection of the corneal tissue. Eight donor corneas were dissected for scRNAseq. First, cells were isolated from six corneas that were manually dissected to separate the epithelial, stromal and the endothelial layers to ensure the gentlest possible enzymatic dissociation of each layer. The corneas were vacuum fixed in a punch base (e.janach) endothelial-cell side up, stained with trypan blue solution (0.4\%) for $30 \mathrm{~s}$ and washed with BSS ophthalmic irrigation solution. The corneal endothelium was then gently lifted following the Schwalbe line using a DMEK cleavage hook (e.janach) and fully stripped using angled McPherson tying forceps. The remaining tissue was trephined using a $9.5 \mathrm{~mm} \varnothing$ Barron vacuum punch (Katena) in order to separate the epithelium and stroma from the scleral ring. The remaining two corneas were used for limbus isolation. A surgical scalpel was used to cut the limbus into approximately $1 \times 2 \mathrm{~mm}$ fragments, which were then rinsed with PBS.

Tissue dissociation to single cells. The six manually dissected corneal tissues were enzymatically treated to obtain single cell suspensions. The stripped corneal endothelium was incubated with $2 \mathrm{mg} / \mathrm{mL}$ collagenase (Sigma) solution in human endothelial serum free media (SFM) (Thermo Fisher Scientific) for $1-2 \mathrm{~h}$ at $37^{\circ} \mathrm{C}$ followed by a $10 \mathrm{~min}$ incubation with Accutase (Thermo Fisher Scientific) at $37^{\circ} \mathrm{C}$ to obtain a single cell suspension. The cells were centrifuged for $5 \mathrm{~min}$ at $800 \times g$ and resuspended in $0.5 \mathrm{~mL}$ of human endothelial SFM media.

The corneal epithelium-stroma tissues were treated with $1.5 \mathrm{mg} / \mathrm{mL}$ collagenase and $0.2 \mathrm{mg} / \mathrm{mL}$ bovine testis hyaluronidase (Sigma) in DMEM/F12 (Thermo Fisher Scientific) for approximately $1 \mathrm{~h}$ at $37^{\circ} \mathrm{C}$. The released corneal epithelium was suctioned with a P1000 micropipette and further treated with Accutase for $10 \mathrm{~min}$ at $37^{\circ} \mathrm{C}$ to obtain a single cell suspension of corneal epithelial cells. The cells were centrifuged for $5 \mathrm{~min}$ at $800 \times g$ and resuspended in $0.5 \mathrm{~mL}$ of DMEM/F12. The remaining corneal stroma was treated with $1.5 \mathrm{mg} / \mathrm{mL}$ collagenase and $0.2 \mathrm{mg} / \mathrm{mL}$ bovine testis hyaluronidase in DMEM/F12 for 5-7 h to obtain a single cell suspension of corneal keratocytes. After the incubation, the cells were centrifuged for $5 \mathrm{~min}$ at $800 \times \mathrm{g}$ and resuspended in $0.5 \mathrm{~mL}$ of DMEM/F12 media.

The limbus fragments isolated from the two corneas for limbal isolation were digested with four trypsinization cycles. In each cycle, the limbus fragments were incubated in $10 \mathrm{~mL}$ of $0.05 \%$ trypsin/0.01\% EDTA (Thermo Fisher Scientific) at $37^{\circ} \mathrm{C}$ for $30 \mathrm{~min}$. The trypsin containing dissociated cells were transferred to a $50 \mathrm{~mL}$ centrifuge tube containing $10 \mathrm{~mL}$ DMEM/F12 media and centrifuged for $5 \mathrm{~min}$ at $300 \times g$, after which the cells were resuspended in $0.5 \mathrm{~mL}$ of DMEM/F12 media. The undigested limbal fragments were placed again in $10 \mathrm{~mL}$ of $0.05 \%$ trypsin $/ 0.01 \%$ EDTA for the following trypsinization cycle. The cells isolated from the limbus of the two donor corneas were pooled for the following steps.

Methanol cell fixation. After dissociation, the single cell suspensions were passed through a $100 \mu \mathrm{m} \varnothing$ cell strainer, centrifuged for $5 \mathrm{~min}$ at $300 \times g$ and resuspended in $1 \mathrm{~mL}$ ice-cold PBS to eliminate any medium remnants. Next, the cells were again centrifuged for $5 \mathrm{~min}$ at $300 \times g$ and resuspended in ice-cold PBS at a ratio of $200 \mu \mathrm{L}$ PBS per $1 \times 10^{6}$ cells followed by the dropwise addition of ice-cold methanol, at a ratio of $800 \mu \mathrm{L}$ per $1 \times 10^{6}$ cells. The cells were stored at $-80^{\circ} \mathrm{C}$ until sequencing.

Single-cell RNA sequencing (scRNAseq). Single-cell mRNA sequencing was performed at Single Cell Discoveries according to standard $10 \times$ Genomics 3' V3.1 chemistry protocol. Prior to loading the cells on the $10 \times$ Chromium controller, cells were rehydrated in rehydration buffer. Cells were then counted to assess cell integrity and concentration. Approximately 9000 cells (comprising 3000 cells from each of the three layers) from corneas $01,02,03$ and 04 were separately loaded by layer and cornea. Furthermore, 3000 cells (1000 cells from each of the three layers) from corneas 05 and 06 , and 3000 cells from corneas 07 and 08 (from the limbal samples) were separately loaded by cornea. The resulting sequencing libraries were prepared following a standard $10 \times$ Genomics protocol.

Bioinformatic analysis of scRNA-seq data. BCL files resulting from sequencing were transformed to FASTQ files with $10 \times$ Genomics Cell Ranger mkfastq. FASTQ files were mapped with Cell Ranger count. During sequencing, Read 1 was assigned 28 base pairs, and were used for identification of the Illumina library barcode, cell barcode and UMI. R2 was used to map the human reference genome GRCh38. Filtering of empty barcodes was done in Cell Ranger. The data from all samples were loaded in R (version 3.6.2) and processed using the Seurat package (version 3.2.0) ( $^{56}$. More specifically, cells with at least 1000 UMIs per cell and less than $20 \%$ mitochondrial gene content were retained for analysis. The data of all $10 \times$ libraries was merged and processed together. The merged dataset was normalized for sequencing depth per cell and log-transformed using a scaling factor of 10,000 . The patient effect was corrected using the integration function of Seurat and used for dimensionality reduction and clustering of all cells or cells selected per layer. Cells were clustered using graph-based clustering and the original Louvain algorithm was utilized for modularity optimization. The differentially expressed genes per cluster were calculated using the Wilcoxon rank sum test and used to identify cell types. Putative doublets were computationally identified using scDblFinder (v1.2.0 $)^{57}$.

Primary culture of limbal cells. Human primary limbal cells were harvested from corneal tissue of cadaveric donors (ages ranging from 36 to 79 years) with informed consent. Human limbal cells were cultured as previously described ${ }^{58,59}$. In short, a surgical scalpel was used to cut the limbus of the corneas into approximately $1 \times 2 \mathrm{~mm}$ fragments, which were then rinsed with PBS. The limbus fragments were then incubated in $10 \mathrm{~mL}$ of 
$0.05 \%$ trypsin $/ 0.01 \%$ EDTA (Thermo Fisher Scientific) at $37^{\circ} \mathrm{C}$ for $30 \mathrm{~min}$. The trypsin containing dissociated cells were transferred to a $50 \mathrm{~mL}$ centrifuge tube containing $10 \mathrm{~mL}$ culture media and centrifuged for $5 \mathrm{~min}$ at $300 \times \mathrm{g}$, after which the cells were resuspended in $0.5 \mathrm{~mL}$ of culture media. The undigested limbal fragments were placed again in $10 \mathrm{~mL}$ of $0.05 \%$ trypsin-EDTA for another trypsinization cycle. Culture medium consisted of 2:1 mixture of DMEM/F12 media (Thermo Fisher Scientific) supplemented with 2 mM GlutaMAX (Thermo Fisher Scientific), 10\% fetal bovine serum (Thermo Fisher Scientific), 125 IU/L insulin (Humulin R, Lilly), $0.2 \mathrm{mM}$ adenine (Merck), $1.1 \mu \mathrm{M}$ hydrocortisone (Merck), $8.5 \mathrm{ng} / \mathrm{mL}$ cholera toxin (Sigma), $2 \mathrm{nM}$ triiodothyronine (Sigma), $10 \mathrm{ng} / \mathrm{mL}$ epidermal growth factor (Amsbio), and $100 \mathrm{IU} / \mathrm{mL}$ penicillin-streptomycin (Thermo Fisher Scientific). The limbal cells were plated on a feeder layer of lethally irradiated 3T3-J2 fibroblasts (fibroblast feeder-layer density 40,000 cells $/ \mathrm{cm}^{2}$ ). The 3T3-J2 fibroblast immortalized cells line was a kind gift of Prof. Howard Green (Harvard Medical School, Boston, MA, USA). When confluent, limbal epithelial stem cells were passaged by $0.05 \%$ trypsin/0.01\% EDTA (Thermo Fisher Scientific) treatment and seeded at a density of 15,000 cells $/ \mathrm{cm}^{2}$ in a Nunc chamber slide (Thermo Fisher Scientific).

Immunofluorescence. Two human donor corneas and primary cultured limbal cells were used for immunofluorescence analysis. The corneas were cut in half transversally, embedded in a cryomold containing TissueTek O.C.T. compound, snap frozen in liquid nitrogen, and stored at $-80{ }^{\circ} \mathrm{C}$ until sectioning. For sectioning, $10 \mu \mathrm{m}$ consecutive sections were cut on an adhesive cryofilm type 3C (16UF) using a modified Kawamoto method $^{60}$, to help preserve the morphology of the tissue during sectioning. The sections were left to dry for 10 min prior to use. The corneal sections and the primary limbal cells cultured on a chamber slide were fixed with $4 \%$ paraformaldehyde, permeabilized with $0.1 \%$ Triton X-100 in PBS for 10 min and blocked with $2 \%$ BSA solution in PBS for $1 \mathrm{~h}$ followed by overnight incubation at $4{ }^{\circ} \mathrm{C}$ with primary antibodies diluted in $2 \%$ BSA blocking solution: mouse monoclonal [1F5D3] anti-Ube2c (1:100 dilution; Thermo Fisher Scientific), mouse monoclonal [1F7G5] anti-CKS2 (1:100 dilution; Thermo Fisher Scientific; 37-0300), rabbit polyclonal anti-P63 (p63a) (1:100 dilution; Cell Signaling Technology; 4892), rabbit polyclonal anti-CPVL (1:100 dilution; Thermo Fisher Scientific), rabbit polyclonal anti-HOMER3 (1:250 dilution; Atlas Antibodies), rabbit polyclonal antiCXCL14 (1:200 dilution; Thermo Fisher Scientific), rabbit polyclonal anti-caveolin 1 (1:300 dilution; Abcam; ab2910), rabbit monoclonal [EP1573Y] anti-Stathmin 1 (1:100 dilution; Abcam), and mouse monoclonal [4A4] anti-P63 ( $\triangle$ Np63) (1:100 dilution; Abcam; ab735). The tissue sections and primary limbal cells were washed five times and incubated with secondary antibodies diluted in 2\% BSA blocking solution, goat anti-rabbit A488 (1:300 dilution; Thermo Fisher Scientific) or goat anti-mouse A568 (1:300 dilution; Thermo Fisher Scientific), for $50 \mathrm{~min}$ at ambient temperature in the dark. Cell nuclei were stained with $0.5 \mu \mathrm{g} / \mathrm{mL}$ DAPI for $10 \mathrm{~min}$. The samples were washed five times in PBS, mounted with coverslips with Fluoromount G mounting medium (Thermo Fisher Scientific) and examined on a Nikon Eclipse Ti-E inverted microscope equipped with a X-Light V2-TP spinning disk (Crest Optics).

\section{Data availability}

The scRNAseq dataset presented in this study has been uploaded to the Gene Expression Omnibus (GEO accession: GSE186433) and can be accessed using this link: https://www.ncbi.nlm.nih.gov/geo/query/acc.cgi?acc= GSE186433. All the datasets presented in this study are available in DataVerseNL and can be accessed using this link: https://doi.org/10.34894/X7ZSDZ.

Received: 9 July 2021; Accepted: 21 October 2021

Published online: 05 November 2021

\section{References}

1. Price, M. O., Mehta, J. S., Jurkunas, U. V. \& Price, F. W. Corneal endothelial dysfunction: Evolving understanding and treatment options. Prog. Retin. Eye Res. 82, 100904 (2021).

2. Pellegrini, G., Rama, P., Di Rocco, A., Panaras, A. \& De Luca, M. Concise review: Hurdles in a successful example of limbal stem cell-based regenerative medicine. Stem Cells 32, 26-34 (2014).

3. Català, P. et al. Approaches for corneal endothelium regenerative medicine. Prog. Retin. Eye Res. (2021) (in press).

4. Ramos, T., Scott, D. \& Ahmad, S. An update on ocular surface epithelial stem cells: Cornea and conjunctiva. Stem Cells Int. 2015, 601731 (2015).

5. Foster, J. W., Gouveia, R. M. \& Connon, C. J. Low-glucose enhances keratocyte-characteristic phenotype from corneal stromal cells in serum-free conditions. Sci. Rep. 5, 10839 (2015).

6. Van den Bogerd, B. et al. Corneal endothelial cells over the past decade: Are we missing the mark(er)?. Transl. Vis. Sci. Technol. 8 , 13 (2019).

7. Fernández-Pérez, J. \& Ahearne, M. Influence of biochemical cues in human corneal stromal cell phenotype. Curr. Eye Res. 44, 135-146 (2019).

8. Pei, Y., Reins, R. Y. \& McDermott, A. M. Aldehyde dehydrogenase (ALDH) 3A1 expression by the human keratocyte and its repair phenotypes. Exp. Eye Res. 83, 1063-1073 (2006).

9. Hayashi, R. et al. Co-ordinated ocular development from human iPS cells and recovery of corneal function. Nature 531, 376-380 (2016).

10. Ouyang, H. et al. WNT7A and PAX6 define corneal epithelium homeostasis and pathogenesis. Nature 511, 358-361 (2014).

11. Van Velthoven, A. J. H. et al. Increased cell survival of human primary conjunctival stem cells in dimethyl sulfoxide-based cryopreservation media. Biopreserv. Biobank. 19, 67-72 (2021).

12. Ramirez-Miranda, A., Nakatsu, M. N., Zarei-Ghanavati, S., Nguyen, C. V. \& Deng, S. X. Keratin 13 is a more specific marker of conjunctival epithelium than keratin 19. Mol. Vis. 17, 1652-1661 (2011).

13. Eghtedari, Y. et al. Keratin 14 expression in epithelial progenitor cells of the developing human cornea. Stem Cells Dev. 25, 699-711 (2016).

14. Cheng, C. C., Wang, D. Y., Kao, M. H. \& Chen, J. K. The growth-promoting effect of KGF on limbal epithelial cells is mediated by upregulation of $\Delta \mathrm{Np} 63 a$ through the p38 pathway. J. Cell Sci. 122, 4473-4480 (2009). 
15. Nasser, W. et al. Corneal-committed cells restore the stem cell pool and tissue boundary following injury. Cell Rep. 22, 323-331 (2018).

16. Yoshida, S. et al. Cytokeratin 15 can be used to identify the limbal phenotype in normal and diseased ocular surfaces. Investig. Ophthalmol. Vis. Sci. 47, 4780-4786 (2006).

17. Li, J. et al. S100A expression in normal corneal-limbal epithelial cells and ocular surface squamous cell carcinoma tissue. Mol. Vis. 17, 2263-2271 (2011).

18. Shurman, D. L., Glazewski, L., Gumpert, A., Zieske, J. D. \& Richard, G. In vivo and in vitro expression of connexins in the human corneal epithelium. Investig. Ophthalmol. Vis. Sci. 46, 1957-1965 (2005).

19. Stepp, M. A. Corneal integrins and their functions. Exp. Eye Res. 83, 3-15 (2006).

20. Schlötzer-Schrehardt, U. \& Kruse, F. E. Identification and characterization of limbal stem cells. Exp. Eye Res. 81, 247-264 (2005).

21. Sun, X. \& Kaufman, P. D. Ki-67: More than a proliferation marker. Chromosoma 127, 175-186 (2018).

22. Wang, C., Zheng, X., Shen, C. \& Shi, Y. MicroRNA-203 suppresses cell proliferation and migration by targeting BIRC5 and LASP1 in human triple-negative breast cancer cells. J. Exp. Clin. Cancer Res. 31, 1-8 (2012).

23. Andäng, M. et al. Histone H2AX-dependent GABAA receptor regulation of stem cell proliferation. Nature 451, 460-464 (2008).

24. Bojic, S. et al. CD200 expression marks a population of quiescent limbal epithelial stem cells with holoclone forming ability. Stem Cells 36, 1723-1735 (2018).

25. Ksander, B. R. et al. ABCB5 is a limbal stem cell gene required for corneal development and repair. Nature 511, 353-357 (2014).

26. de Paiva, C. S., Chen, Z., Corrales, R. M., Pflugfelder, S. C. \& Li, D. ABCG2 transporter identifies a population of clonogenic human limbal epithelial cells. Stem Cells 23, 63-73 (2005).

27. Bonnet, C. et al. Human limbal epithelial stem cell regulation, bioengineering and function. Prog. Retin. Eye Res. https://doi.org/ 10.1016/j.preteyeres.2021.100956 (2021).

28. He, J. et al. Tissue engineered corneal epithelium derived from clinical-grade human embryonic stem cells. Ocul. Surf. 18, 672-680 (2020).

29. Kitazawa, K. et al. PAX6 regulates human corneal epithelium cell identity. Exp. Eye Res. 154, 30-38 (2017).

30. Hodge, R. G. \& Ridley, A. J. Regulation and functions of RhoU and RhoV. Small GTPases 11, 8-15 (2020).

31. Yoshida, Y., Ban, Y. \& Kinoshita, S. Tight junction transmembrane protein claudin subtype expression and distribution in human corneal and conjunctival epithelium. Investig. Ophthalmol. Vis. Sci. 50, 2103-2108 (2009).

32. $\mathrm{Li}$, X. et al. CD44 is involved in SMAD signaling by TGFb in keratocytes. Investig. Ophthalmol. Vis. Sci. 50, 4539 (2009).

33. Choong, P. F., Mok, P. L., Cheong, S. K. \& Then, K. Y. Mesenchymal stromal cell-like characteristics of corneal keratocytes. Cytotherapy $9,252-258$ (2007).

34. Acharya, P. S. et al. Fibroblast migration is mediated by CD44-dependent TGF $\beta$ activation. J. Cell Sci. 121, 1393-1402 (2008).

35. Jester, J. V. et al. Myofibroblast differentiation of normal human keratocytes and hTERT, extended-life human corneal fibroblasts. Investig. Ophthalmol. Vis. Sci. 44, 1850-1858 (2003).

36. Wilson, S. E. Corneal myofibroblasts and fibrosis. Exp. Eye Res. 201, 108272 (2020).

37. Ding, V., Chin, A., Peh, A., Mehta, J. S. \& Choo, A. Generation of novel monoclonal antibodies for the enrichment and characterization of human corneal endothelial cells (hCENC) necessary for the treatment of corneal endothelial blindness. MAbs 6, 1439-1452 (2014).

38. Okumura, N. et al. Cell surface markers of functional phenotypic corneal endothelial cells. Investig. Ophthalmol. Vis. Sci. 55, 7610-7618 (2014).

39. Ning, W. et al. The CAMSAP3-ACF7 complex couples noncentrosomal microtubules with actin filaments to coordinate their dynamics. Dev. Cell 39, 61-74 (2016).

40. Chen, L. et al. Ap-2 $\beta$ is a downstream effector of PITX2 required to specify endothelium and establish angiogenic privilege during corneal development. Investig. Ophthalmol. Vis. Sci. 57, 1072-1081 (2016).

41. Kumar, S. \& Duester, G. Retinoic acid signaling in perioptic mesenchyme represses Wnt signaling via induction of Pitx2 and Dkk2. Dev. Biol. 340, 67-74 (2010).

42. Collin, J. et al. A single cell atlas of human cornea that defines its development, limbal progenitor cells and their interactions with the immune cells. Ocul. Surf. 21, 279-298 (2021).

43. Li, D. Q. et al. Single-cell transcriptomics identifies limbal stem cell population and cell types mapping its differentiation trajectory in limbal basal epithelium of human cornea. Ocul. Surf. 20, 20-32 (2021).

44. Dou, S. et al. Molecular identity of human limbal heterogeneity involved in corneal homeostasis and privilege: Single-cell RNA sequencing of human limbus. Ocul. Surf. 21, 206-220 (2021).

45. Li, J.-M. et al. Single-cell transcriptomics identifies a unique entity and signature markers of transit-amplifying cells in human corneal limbus. Investig. Opthalmol. Vis. Sci. 62, 36 (2021).

46. Kaplan, N. et al. Single-cell RNA transcriptome helps define the limbal/corneal epithelial stem/early transit amplifying cells and how autophagy affects this population. Investig. Ophthalmol. Vis. Sci. 60, 3570-3583 (2019).

47. Guindolet, D. et al. Epithelial regeneration in human corneas preserved in an active storage machine. Transl. Vis. Sci. Technol. 10, $31(2021)$.

48. Du, Y., Funderburgh, M. L., Mann, M. M., SundarRaj, N. \& Funderburgh, J. L. Multipotent stem cells in human corneal stroma. Stem Cells 23, 1266-1275 (2005).

49. Du, Y. et al. Secretion and organization of a cornea-like tissue in vitro by stem cells from human corneal stroma. Investig. Ophthalmol. Vis. Sci. 48, 5038-5045 (2007).

50. Wu, J., Du, Y., Mann, M. M., Funderburgh, J. L. \& Wagner, W. R. Corneal stromal stem cells versus corneal fibroblasts in generating structurally appropriate corneal stromal tissue. Exp. Eye Res. 120, 71-81 (2014).

51. Cheong, Y. K. et al. Identification of cell surface markers glypican-4 and CD200 that differentiate human corneal endothelium from stromal fibroblasts. Investig. Ophthalmol. Vis. Sci. 54, 4538-4547 (2013).

52. Yam, G. H. et al. Characterization of human transition zone reveals a putative progenitor-enriched niche of corneal endothelium. Cells 8, 1244 (2019).

53. Van den Bogerd, B., Dhubhghaill, S. N., Koppen, C., Tassignon, M. J. \& Zakaria, N. A review of the evidence for in vivo corneal endothelial regeneration. Surv. Ophthalmol. 63, 149-165 (2018).

54. Mattei, D. et al. Enzymatic dissociation induces transcriptional and proteotype bias in brain cell populations. Int. J. Mol. Sci. 21, 7944 (2020).

55. Denisenko, E. et al. Systematic assessment of tissue dissociation and storage biases in single-cell and single-nucleus RNA-seq workflows. Genome Biol. 21, 130 (2020).

56. Stuart, T. et al. Comprehensive integration of single-cell data. Cell 177, 1888-1902 (2019).

57. Germain, P.-L. scDblFinder: scDblFinder. R package version 1.2.0. (2020).

58. Pellegrini, G. et al. Long-term restoration of damaged corneal surfaces with autologous cultivated corneal epithelium. Lancet 349, 990-993 (1997).

59. Rama, P. et al. Limbal stem-cell therapy and long-term corneal regeneration. N. Engl. J. Med. 363, 147-155 (2010).

60. Kawamoto, T. \& Kawamoto, K. Preparation of thin frozen sections from nonfixed and undecalcified hard tissues using Kawamoto's film method. Methods Mol. Biol. 2230, 259-281 (2021). 


\section{Acknowledgements}

The authors thank Annika Jeschke and Timo Rademakers (Maastricht University) for the assistance in the immunofluorescence experiments and the sample cryosectioning. The authors thank Single Cell Discoveries (Utrecht, the Netherlands) for the single cell sequencing services provided. The authors thank ETB-BISLIFE: Multi Tissue Center and Saving Sight Kansas City for providing research-grade human corneas.

\section{Author contributions}

P.C.: experimental design, tissue acquisition, tissue dissection and dissociation to single cells, microscopy imaging for immunofluorescence experiments, experimental data analysis, manuscript and figures preparation. N.G.: bioinformatics analysis, data collection, manuscript and figures preparation. J.A.D.: experimental data analysis. E.S.: experimental design, tissue acquisition, tissue dissection and dissociation to single cells, experimental data analysis. A.J.H.v.V.: limbal tissue dissection and dissociation to single cells, immunofluorescence experiments, manuscript preparation. V.L.S.L., M.M.D., and R.M.M.A.N.: fund acquisition, experimental design, tissue acquisition, manuscript and figures preparation, and supervised the work. All authors ensured that questions on the accuracy or integrity of all parts of the study were appropriately researched and resolved.

\section{Funding}

This research was funded by Chemelot InSciTe under the EyeSciTe consortium and ZonMw Enabling Technology Hotels grant 435005012.

\section{Competing interests}

The authors declare no competing interests.

\section{Additional information}

Supplementary Information The online version contains supplementary material available at https://doi.org/ 10.1038/s41598-021-01015-w.

Correspondence and requests for materials should be addressed to M.M.D. or V.L.S.L.

Reprints and permissions information is available at www.nature.com/reprints.

Publisher's note Springer Nature remains neutral with regard to jurisdictional claims in published maps and institutional affiliations.

(c) (1) Open Access This article is licensed under a Creative Commons Attribution 4.0 International License, which permits use, sharing, adaptation, distribution and reproduction in any medium or format, as long as you give appropriate credit to the original author(s) and the source, provide a link to the Creative Commons licence, and indicate if changes were made. The images or other third party material in this article are included in the article's Creative Commons licence, unless indicated otherwise in a credit line to the material. If material is not included in the article's Creative Commons licence and your intended use is not permitted by statutory regulation or exceeds the permitted use, you will need to obtain permission directly from the copyright holder. To view a copy of this licence, visit http://creativecommons.org/licenses/by/4.0/.

(c) The Author(s) 2021 